\title{
Static behaviour of induced seismicity
}

\author{
Arnaud Mignan \\ Institute of Geophysics, ETH Zurich, Zurich, Switzerland \\ Correspondence to: Arnaud Mignan (arnaud.mignan@sed.ethz.ch)
}

Received: 6 October 2015 - Published in Nonlin. Processes Geophys. Discuss.: 10 December 2015

Revised: 15 February 2016 - Accepted: 13 April 2016 - Published: 29 April 2016

\begin{abstract}
The standard paradigm to describe seismicity induced by fluid injection is to apply non-linear diffusion dynamics in a poroelastic medium. I show that the spatiotemporal behaviour and rate evolution of induced seismicity can, instead, be expressed by geometric operations on a static stress field produced by volume change at depth. I obtain laws similar in form to the ones derived from poroelasticity while requiring a lower description length. Although fluid flow is known to occur in the ground, it is not pertinent to the geometrical description of the spatio-temporal patterns of induced seismicity. The proposed model is equivalent to the static stress model for tectonic foreshocks generated by the Non-Critical Precursory Accelerating Seismicity Theory. This study hence verifies the explanatory power of this theory outside of its original scope and provides an alternative physical approach to poroelasticity for the modelling of induced seismicity. The applicability of the proposed geometrical approach is illustrated for the case of the 2006, Basel enhanced geothermal system stimulation experiment. Applicability to more problematic cases where the stress field may be spatially heterogeneous is also discussed.
\end{abstract}

\section{Introduction}

Induced seismicity is a growing concern for the energy industry relying on fluid injection in the deep parts of the Earth's crust (Ellsworth, 2013; Mignan et al., 2015). At the same time, fluid injection sites provide natural laboratories to study the impact of increased fluid pressure on earthquake generation (Majer et al., 2007). Induced seismicity is characterised by two empirical laws, namely (i) a linear relationship between the fluid mass $m(t)$ injected up to time $t$ and the cumulative number of induced earthquakes $N(t)$ and (ii) a parabolic induced seismicity spatial envelope ra- dius $r \propto \sqrt[n]{m(t)}$, with $n$ being a positive integer (Shapiro and Dinske, 2009). These two simple descriptive laws can be derived from the differential equations of poroelasticity (Biot, 1941) under various assumptions (Shapiro and Dinske, 2009). In general however, the full description of the process requires complex numeric modelling coupling fluid flow, heat transport, and geomechanics (Rutqvist, 2011). These models, numerically cumbersome, can become intractable because of the sheer number of parameters (Miller, 2015). Attempts to additionally correct for the known discrepancies between Biot's theory and rock experiments have led to a large variety of model assumptions (Berryman and Wang, 2001), indicating that poroelasticity results are ambiguous.

I will demonstrate that a static stress model can explain the two empirical laws of induced seismicity without requiring any concept of poroelasticity. The proposed theoretical framework hence avoids the aforementioned shortcomings by suggesting an origin of induced seismicity that does not involve fluid flow in a porous medium (although fluid flow indeed occurs). Historically, a similar static stress model was proposed for the tectonic regime under the NonCritical Precursory Accelerating Seismicity Theory (N-C PAST) (Mignan et al., 2007; Mignan, 2008, 2012). Its application to induced seismicity data will allow a more fundamental investigation of the relationship between static stress and earthquake generation. To test the model, I will use data from the 2006 Basel enhanced geothermal system (EGS) stimulation experiment, including the flow rate of injected fluids (Häring et al., 2008) and the relocated catalogue of induced seismicity (Kraft and Deichmann, 2014). 


\section{The Non-Critical Precursory Accelerating Seismicity Theory (N-C PAST)}

The N-C PAST has been proposed to explain the precursory seismicity patterns observed before large earthquakes from geometric operations in the spatio-temporal stress field generated by constant tectonic stress accumulation (Mignan et al., 2007; Mignan, 2008, 2012). In particular, it provides a physical algebraic expression of temporal power laws without requiring local interactions between the elements of the system (Sammis and Sornette, 2002; Mignan, 2011). Therefore earthquakes are considered passive (static) tracers of the stress accumulation process, in contrast with active earthquake cascading in a critical process (hence the term "noncritical"). The concept of self-organised criticality (Bak and Tang, 1989) is seldom used to explain induced seismicity (Grasso and Sornette, 1998). Since there is no equivalent of a mainshock in induced seismicity, the criticality-versus-noncriticality debate has limited meaning in that case. However, the underlying process of static stress changes considered in the N-C PAST can be tested against the observed spatiotemporal behaviour of induced seismicity.

The N-C PAST postulates that earthquake activity can be categorised into three regimes - background, quiescence, and activation - depending on the spatio-temporal stress field $\sigma(r, t)$ :

$\sigma(r, t)=\left\{\begin{aligned} \sigma_{0}^{*}, & t<t_{0} \\ \frac{h^{n}}{\left(r^{2}+h^{2}\right)^{\frac{n}{2}}}\left(\sigma_{0}+\dot{\tau}\left(t-t_{0}\right)\right)+\sigma_{0}^{*}, & t_{0} \leq t<t_{\mathrm{f}}\end{aligned}\right.$

defined from the boundary conditions $\sigma(r+\infty, t)=\sigma_{0}^{*}$ and $\sigma(r=0, t)=\sigma_{0}+\dot{\tau} t+\sigma_{0}^{*}$, with $h$ being the depth of the fault segment base, $r$ the distance along the stress field gradient from the fault's surface projection, $\sigma_{0}<0$ the stress drop associated with a hypothetical silent slip occurring at $t_{0}$ at the base of the fault, $\dot{\tau}$ the tectonic stress rate on the fault, $\sigma_{0}^{*}$ the crustal background stress, $n=3$ the spatial diffusion exponent for static stress, and $t_{\mathrm{f}}$ the mainshock occurrence time (Mignan et al., 2007) (Fig. 1a). Background, quiescence, and activation regimes are strictly defined by the three event spatio-temporal densities $\delta_{\mathrm{b} 0}, \delta_{\mathrm{bm}}$, and $\delta_{\mathrm{bp}}$ for $|\sigma| \leq \sigma_{0}^{*} \pm \Delta \sigma^{*}, \sigma<\sigma_{0}^{*}-\Delta \sigma^{*}$, and $\sigma>\sigma_{0}^{*}+\Delta \sigma^{*}$, respectively, with the boundary layer being $\pm \Delta \sigma^{*}$ the background stress amplitude range (so-called N-C PAST postulate). By definition, $\delta_{\mathrm{bm}}<\delta_{\mathrm{b} 0}<\delta_{\mathrm{bp}}$, with each seismicity regime so far being assumed isotropic and homogeneous in space (i.e. the role of the fault network is neglected). Correlation between earthquake productivity and static stress changes is well established (King, 2007). The distinction of three unique seismicity regimes with constant event density, the main assumption of the N-C PAST, is discussed later on.

In the tectonic case, static stress changes are underloading due to hypothetical precursory silent slip on the fault at $t_{0}$ followed by overloading due to hypothetical asperities delaying rupture on the fault after $t_{\mathrm{p}}^{*}$ (Mignan, 2012). The three
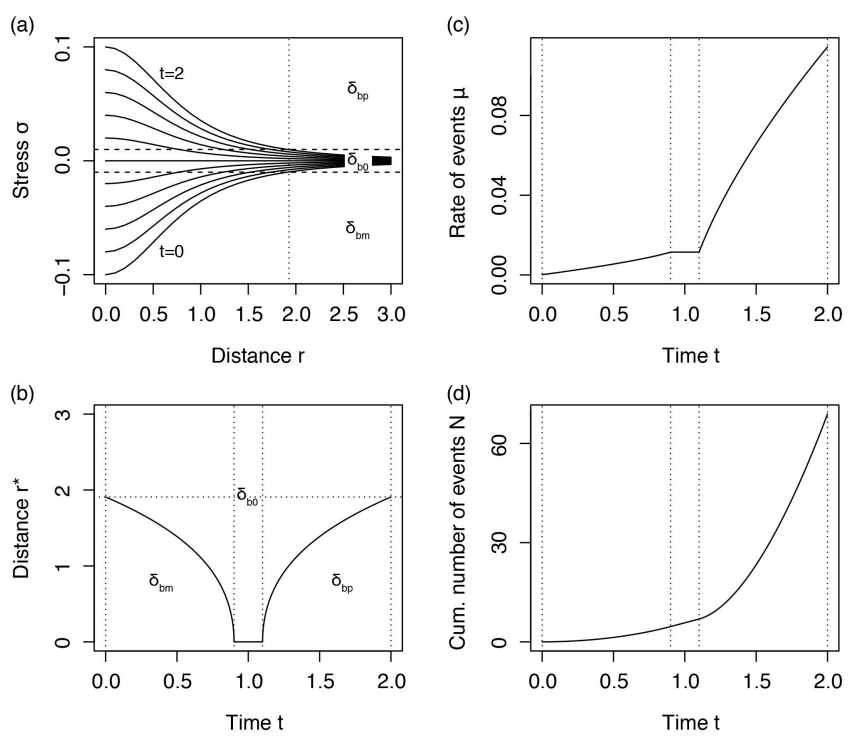

Figure 1. Seismicity spatio-temporal behaviour described by the N-C PAST static stress model (tectonic case; Mignan, 2012): (a) spatio-temporal evolution of the stress field $\sigma(r, t)$ generated by constant stress accumulation $\dot{\tau}$ on a fault located at $r=0$ (Eq. 1). Background, quiescence, and activation seismicity regimes are described by densities of events $\delta_{\mathrm{b} 0}, \delta_{\mathrm{bm}}$, and $\delta_{\mathrm{bp}}$ for $|\sigma| \leq \sigma_{0}^{*} \pm \Delta \sigma^{*}$, $\sigma<\sigma_{0}^{*}-\Delta \sigma^{*}$, and $\sigma>\sigma_{0}^{*}+\Delta \sigma^{*}$, respectively; (b) temporal evolution of quiescence and activation envelopes $r^{*}(t)$, with $\sigma\left(r^{*}\right)=$ $\sigma_{0}^{*} \pm \Delta \sigma^{*}$ (Eq. 2); (c) rate of events $\mu(t)$ in a disc of constant radius $\max \left(r^{*}\right)$ (Eq. 3); (d) cumulative number of events $N(t)$ (Eq. 4 ) of power law form (Eq. 5), with $t_{0}=0, t_{\mathrm{mid}}=1, t_{\mathrm{f}}=2, h=1, \dot{\tau}=0.1$, $\sigma_{0}^{*}=0, \Delta \sigma^{*}=10^{-2}, \delta_{\mathrm{bm}}=0.001, \delta_{\mathrm{b} 0}=0.1, \delta_{\mathrm{bp}}=1, n=3, k=$ $\pi, d=2, \Delta t=0.01$.

seismicity regimes are then defined as solid spatio-temporal objects with envelopes

$$
\left\{\begin{array}{l}
r_{\mathrm{Q}}^{*}\left(t_{0} \leq t<t_{\mathrm{m}}^{*}\right)=h\left[\left(\frac{\dot{\tau}\left(t_{\mathrm{m}}^{*}-t\right)}{\Delta \sigma^{*}}+1\right)^{2 / n}-1\right]^{1 / 2} \\
r_{\mathrm{A}}^{*}\left(t_{\mathrm{p}}^{*}<t<t_{\mathrm{f}}\right)=h\left[\left(\frac{\dot{\tau}\left(t-t_{\mathrm{p}}^{*}\right)}{\Delta} \sigma^{*}+1\right)^{2 / n}-1\right]^{1 / 2}
\end{array}\right.
$$

by applying to Eq. (1) the boundary conditions $\sigma\left(r_{\mathrm{Q}}^{*}, t\right)=$ $\sigma\left(0, t_{\mathrm{m}}^{*}\right)=\sigma_{0}^{*}-\Delta \sigma^{*}$ and $\sigma\left(r_{\mathrm{A}}^{*}, t\right)=\sigma\left(0, t_{\mathrm{p}}^{*}\right)=\sigma_{0}^{*}+\Delta \sigma^{*}$, respectively. The parameters $t_{\mathrm{m}}^{*}=t_{\mathrm{mid}}-\Delta \sigma^{*} / \dot{\tau}$ and $t_{\mathrm{p}}^{*}=$ $t_{\text {mid }}+\Delta \sigma^{*} / \dot{\tau}$ represent the times of quiescence disappearance and of activation appearance, respectively, with $\sigma\left(0, t_{\mathrm{mid}}\right)=\sigma_{0}^{*}$. The background seismicity regime is defined by subtracting the quiescence and activation envelopes $r_{\mathrm{A}}^{*}(t)$ and $r_{\mathrm{Q}}^{*}(t)$ from a larger constant envelope $r_{\max } \geq$ $\max \left(r_{\mathrm{A}}^{*}, r_{\mathrm{Q}}^{*}\right)$ to avoid truncating the quiescence and activation solids (Fig. 1b). While trivial along $\boldsymbol{r}$, concepts of geometric modelling may be required to represent these seis- 
micity solids in three-dimensional Euclidian space (Gallier, 1999 ) in which the vector $\boldsymbol{r}$ may change direction in space (Mignan, 2008, 2011). The non-stationary background seismicity rate $\mu(t)$ is then defined in the volume of maximum extent $r_{\max }$ by

$\mu(t)= \begin{cases}\delta_{\mathrm{b} 0} k r_{\max }^{d}, & t<t_{0} \\ \delta_{\mathrm{b} 0} k\left(r_{\max }^{d}-r_{\mathrm{Q}}^{*}(t)^{d}\right)+\delta_{\mathrm{bm}} k r_{\mathrm{Q}}^{*}(t)^{d}, & t_{0} \leq t<t_{\mathrm{m}}^{*} \\ \delta_{\mathrm{b} 0} k r_{\max }^{d}, & t_{\mathrm{m}}^{*} \leq t \leq t_{\mathrm{p}}^{*} \\ \delta_{\mathrm{b} 0} k\left(r_{\max }^{d}-r_{\mathrm{A}}^{*}(t)^{d}\right)+\delta_{\mathrm{bp}} k r_{\mathrm{A}}^{*}(t)^{d}, & t_{\mathrm{p}}^{*}<t<t_{\mathrm{f}}\end{cases}$

with $k$ being a geometric parameter and $d$ the spatial dimension. For the tectonic case in which $r_{\max } \gg h$, the volume is assumed to be a cylinder with $k=\pi, d=2$, and $\delta$ being the density of epicentres in space (Fig. 1c). It should be noted that taking $r_{\max }$ very large relative to $\max \left(r_{\mathrm{A}}^{*}, r_{\mathrm{Q}}^{*}\right)$ tends to mask the non-stationary seismicity pattern to be investigated. As a consequence it is preferable in practice to use $r_{\max }=\max \left(r_{\mathrm{A}}^{*}, r_{\mathrm{Q}}^{*}\right)$. Finally, the cumulative number of events $N(t)$ is defined as

$N(t)=\int_{0}^{t_{\mathrm{f}}} \mu(t) \mathrm{d} t$,

which represents a power law time-to-failure equation of the form

$N(t) \propto t+t^{\frac{d}{n}+1}$,

with the first term representing the linear background seismicity and the second term the quiescence or activation power law behaviour observed prior to some large mainshocks (Fig. 1d) (see the review by Sammis and Sornette (2002) for different physical processes yielding a temporal power law).

\section{Application of the N-C PAST static stress model to induced seismicity}

In the case of an EGS stimulation, the stress source is the fluid injected at depth with overpressure

$P(t, r=0)=K \frac{\Delta V(t, \Delta t)}{V_{0}}$,

where $K$ is the bulk modulus, $\Delta V$ the volume change per time unit, and $V_{0}$ the infinitesimal volume subjected to pressure effect per time unit at the borehole located at $r=0$. The injected volume $V(t)$ is determined from the flow rate profile $Q(t)$, as

$V(t)=\int_{t_{0}}^{t} Q(t) \mathrm{d} t$, with $t_{0}$ being the starting time of the injection. The volume change rate is then defined as

$\Delta V(t, \Delta t)=\frac{V(t)-V(t-\Delta t)}{\Delta t}$,

with $\Delta t$ being a time increment.

In the EGS case, $r \cong h$, with $h$ being the borehole depth and induced seismicity defined as hypocentres. The spatiotemporal stress field $\sigma(r, t)$ becomes

$\sigma(r, t)= \begin{cases}\sigma_{0}^{*}, & t<t_{0} \\ \frac{r_{0}^{n}}{\left(r+r_{0}\right)^{n}} P(t, r=0)+\sigma_{0}^{*}, & t \geq t_{0}\end{cases}$

with $r$ being the distance along the stress field gradient from the borehole; $n=3$ the spatial diffusion exponent for static stress; and $r_{0} \rightarrow 0$ the infinitesimal radius of volume $V_{0}=$ $k r_{0}^{d} / t_{0}$, where $t_{0}=1$ is the time unit. The parameter $r_{0}$ is incidental and disappears in the induced seismicity case (see below). Activation represents the case when fluids are injected and quiescence when fluids are ejected (bleed-off), or, in terms of stress field variations, when the pressure change by fluid injection is positive or negative, respectively. It follows that

$\left\{\begin{array}{l}r_{\mathrm{A}}^{*}(t \mid \Delta V \geq 0)=\left(\frac{r_{0}^{n-d}}{k} \frac{K t_{0}}{\Delta \sigma^{*}} \Delta V(t)\right)^{1 / n}-r_{0} \\ r_{\mathrm{Q}}^{*}(t \mid \Delta V<0)=\left(-\frac{r_{0}^{n-d}}{k} \frac{K t_{0}}{\Delta \sigma^{*}} \Delta V(t)\right)^{1 / n}-r_{0},\end{array}\right.$

which suggests that the spatio-temporal shape of the induced seismicity envelope depends on the $n$th root of the flow rate profile $Q(t)$ (with $n=3$ in the static stress case). This parabolic relationship is similar to the generalised form $r(t) \propto m(t)^{1 / d}$ derived from non-linear poroelasticity in a heterogeneous medium where $m$ is the cumulative mass of injected fluid and $d$ the spatial dimension (Shapiro and Dinske, 2009). The main difference between the two physical approaches is in the underlying stress field, which is here static and in poroelasticity dynamic and related to the displacement gradient of the fluid mass (Rudnicki, 1986). As a side note, it is trivial to derive Eq. (10) from Eq. (9), while numerous assumptions are necessary to obtain the parabolic form $m(t)^{1 / d}$ in non-linear poroelasticity (Shapiro and Dinske, 2009).

The induced seismicity rate $\mu(t)$ is then defined by Eq. (3) but with $r^{*}$ from Eq. (10), $k=4 \pi / 3$, and $d=3$, assuming a spherical spatial volume (i.e. isotropic stress field). For the activation phase (i.e. stimulation period), it follows that

$N(t) \propto \Delta V(t)^{\frac{d}{n}+1}$

or

$N(t) \propto V(t)^{\frac{d}{n}}$. 
The induced seismicity case $d=n=3$ confirms the linear relationship between cumulative injected volume and cumulative number of induced earthquakes $N(t) \propto V(t)$ previously derived from poroelasticity (e.g. Shapiro and Dinske, 2009). In contrast with poroelasticity, this second law is a direct consequence of the first. The $d=n$ condition also yields the simplified form of Eq. (10):

$$
\left\{\begin{array}{l}
r_{\mathrm{A}}^{*}(t \mid \Delta V \geq 0) \approx\left(\frac{3}{4 \pi} \frac{K t_{0}}{\Delta \sigma^{*}} \Delta V(t)\right)^{1 / 3} \\
r_{\mathrm{Q}}^{*}(t \mid \Delta V<0) \approx\left(-\frac{3}{4 \pi} \frac{K t_{0}}{\Delta \sigma^{*}} \Delta V(t)\right)^{1 / 3},
\end{array}\right.
$$

where the one free parameter is the normalised background stress amplitude range $\widehat{\Delta \sigma^{*}}=\Delta \sigma^{*} /\left(K t_{0}\right)$.

\section{Application to the 2006 Basel EGS induced seismicity sequence}

Figure 2 shows the flow rate $Q(t)$ of injected fluids during the 2006 Basel EGS stimulation experiment (Häring et al., 2008) and the spatio-temporal distribution of relocated induced seismicity (Kraft and Deichmann, 2014) above completeness magnitude $M_{\mathrm{c}}=0.8$. The injection started at 18:00 LT on 2 December $2006\left(t_{0}\right)$ and stopped at 11:33 LT on 8 December $2006\left(t_{1}\right)$, after which the well was bled off $(\Delta V<0)$ (Fig. 2a). The N-C PAST thus predicts an activation envelope $r_{\mathrm{A}}^{*}$ for $t_{0} \leq t<t_{1}$ and a quiescence envelope $r_{\mathrm{Q}}^{*}$ for $t \geq t_{1}$ (Eq. 13). The activation and quiescence envelopes are fitted to the Basel data using $\widehat{\Delta \sigma^{*}} \in\left[10^{-3}, 10^{-1}\right]$ day $^{-1}$ (light curves) and $\Delta t=1 / 4$ day. The results are shown in Fig. 2b. The value $\widehat{\Delta \sigma^{*}}=0.007 \mathrm{day}^{-1}$ (dark curves) provides the best fit to the data, defined from the best score $S=\left(w_{\mathrm{A}}+w_{\mathrm{Q}}\right) / 2$, with $w_{\mathrm{A}}$ and $w_{\mathrm{Q}}$ being the ratio of events of distance $r \leq r_{\mathrm{A}}^{*}$ and $r \geq r_{\mathrm{Q}}^{*}$ in the injection and bleedingoff phases, respectively. Figure 2c shows $S$ as a function of $\widehat{\Delta \sigma^{*}}$ for $\Delta t=\{1 / 12,1 / 8,1 / 4\}$ day, which indicates that the results remain stable for lower time increments.

I evaluate $\delta_{\mathrm{b} 0}=10^{-10}$ event $^{-3} \mathrm{day}^{-1}$ by counting all earthquakes declared in the national Swiss catalogue (ECOS09, http://hitseddb.ethz.ch:8080/ecos09/) and located within $10 \mathrm{~km}$ of the borehole of coordinates $\left(7.594^{\circ} \mathrm{E} ; 47.586^{\circ} \mathrm{N}\right)$ and depth $4.36 \mathrm{~km}$. This means that $\sim 1$ tectonic earthquake is expected on average in the space-time window considered. Due to the low tectonic activity in the area, I approximate $\delta_{\mathrm{b} 0}=\delta_{\mathrm{bm}}=0$ event $^{-3} \mathrm{day}^{-1}$ (i.e. total quiescence). The theory shows a good agreement with the observations, with $97 \%$ of the seismicity below $r_{\mathrm{A}}^{*}$ during the injection phase (red points in Fig. $2 \mathrm{~b}$ ) and $98 \%$ of the seismicity above $r_{\mathrm{Q}}^{*}$ during the bleeding-off phase (orange to yellow points).

The density of events above $r_{\mathrm{Q}}^{*}$ is however not $\delta_{\mathrm{b} 0}$ but an equation of the form

$\delta_{\mathrm{b}}\left(t \geq t_{1}\right)=\delta_{\mathrm{bp}} \exp \left(-\frac{t-t_{1}}{\tau}\right)$,
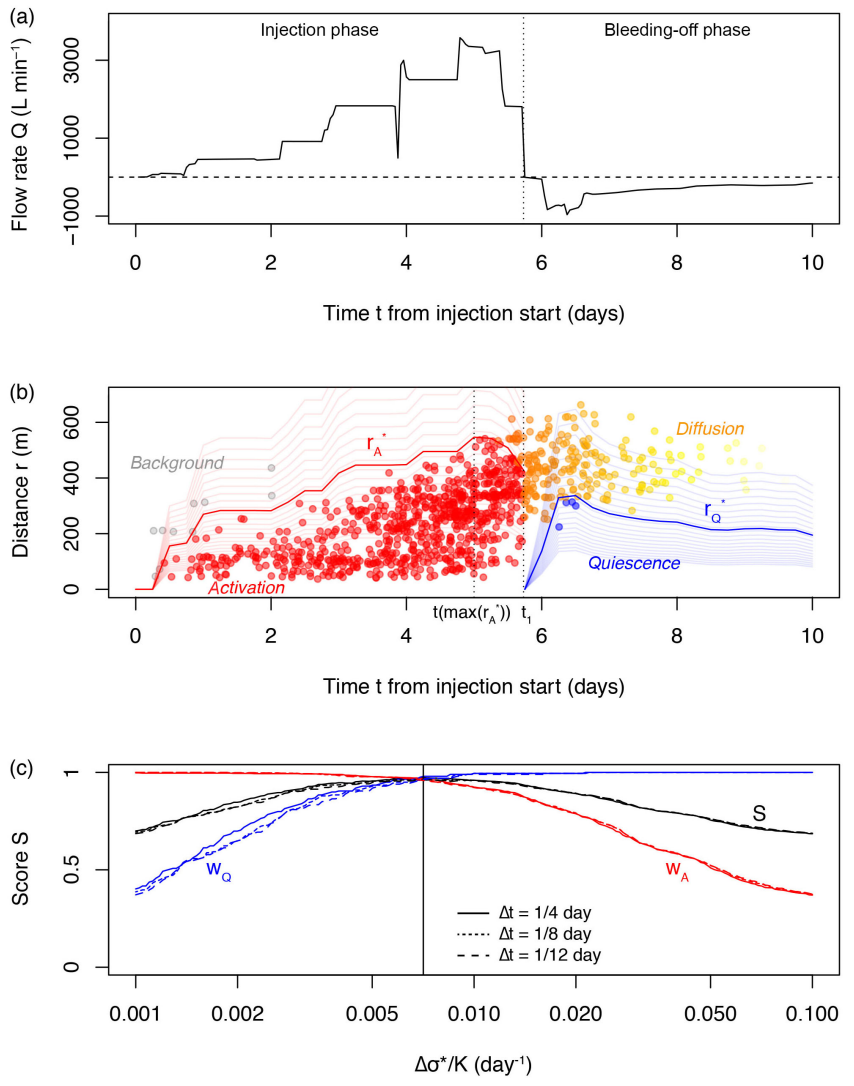

Figure 2. 2006 Basel EGS stimulation experiment data with activation and quiescence envelope fits: (a) flow rate $Q(t)$ (digitised from Häring et al., 2008); (b) spatio-temporal distribution of relocated induced seismicity (Kraft and Deichmann, 2014) with $r$ the distance from the borehole. The activation and quiescence envelopes $r_{\mathrm{A}}^{*}(t)$ and $r_{\mathrm{Q}}^{*}(t)$ are defined from Eq. (13) with parameters $\widehat{\Delta \sigma^{*}}=0.007 \mathrm{day}^{-1}$ (dark curves) and $\Delta t=1 / 4$ day. The light curves represent the range $\widehat{\Delta \sigma^{*}} \in\left[10^{-3}, 10^{-1}\right]$ day $^{-1}$ in 0.1 increments on the $\log _{10}$ scale. Points represent the induced earthquakes; which colour indicates how they are declared. (c) Score $S=\left(w_{\mathrm{A}}+w_{\mathrm{Q}}\right) / 2$, with $w_{\mathrm{A}}$ and $w_{\mathrm{Q}}$ being the ratio of events of distance $r \leq r_{\mathrm{A}}^{*}$ and $r \geq r_{\mathrm{Q}}^{*}$ in the injection and bleeding-off phases, respectively. The vertical line represents $\widehat{\Delta \sigma^{*}}=0.007 \mathrm{day}^{-1}$.

which represents the temporal diffusion of induced seismicity; $\tau$ is the average time constant (e.g. Mignan, 2015). Diffusion from density $\delta_{\mathrm{bp}}$ to $\delta_{\mathrm{b} 0}$ was originally not considered in the N-C PAST as any potential diffusion after an activated foreshock sequence would be shadowed by the effects of the subsequent mainshock. Here however, diffusion dominates in the post-injection phase. Equation (14) represents a relaxation process from the overloading state to the background state. The results here suggest that only the events declared as background (grey points) and quiescence events (blue points) are outliers. The observed variations in $r$ below $r_{\mathrm{A}}^{*}$ and above $r_{\mathrm{O}}^{*}$ are not explained by the model, which only predicts the behaviour of the activation and quiescence 

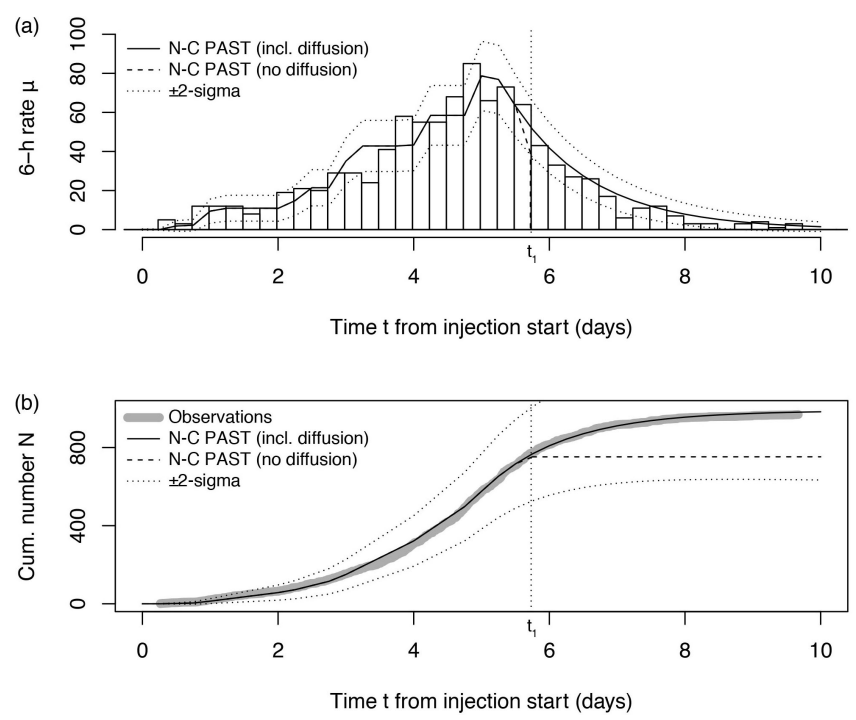

Figure 3. Induced seismicity production time series, observed and predicted: (a) histogram of the observed $6 \mathrm{~h}$ induced seismicity rate $\mu(t)$ with fit based on Eq. (15) with MLE parameters $\delta_{\mathrm{bp}}=4.68 \times$ $10^{-7}$ event $^{-3}$ day $^{-1}$ (production parameter) and $\tau=1.18$ day (diffusion parameter); (b) cumulative number of induced earthquakes $N(t)$ with fit based on Eq. (4) with $\mu(t)$ of Eq. (15).

fronts. The second-order variations may be due to anisotropic effects and for $t>t\left(\max \left(r_{\mathrm{A}}^{*}\right)\right)$ to additional spatial diffusion effects.

Figure 3 shows the $6 \mathrm{~h}$ rate of induced seismicity $\mu(t)$ and the cumulative number of induced events $N(t)$, observed and predicted. With $\delta_{\mathrm{b} 0}=\delta_{\mathrm{bm}}=0$ and taking into account induced seismicity temporal diffusion, the rate of induced seismicity becomes

$$
\begin{aligned}
\mu(t)= & \max \left(\frac{4 \pi}{3} \delta_{\mathrm{bp}} \cdot \Delta t \cdot r^{*}(t)^{3}, \frac{4 \pi}{3} \delta_{\mathrm{bp}} \cdot \Delta t \cdot r^{*}\left(t-S_{t}\right)^{3}\right. \\
& \left.\exp \left(-\frac{t-S_{t}}{\tau}\right)\right)
\end{aligned}
$$

where $\delta_{\text {bp }}=4.68 \times 10^{-7}$ event $^{-3}$ day $^{-1}$ (production parameter) and $\tau=1.18$ day (diffusion parameter) are obtained by maximum-likelihood estimation (MLE), $S_{t}=$ $\{\Delta t, \ldots, i \Delta t, \ldots\}$, and

$r^{*}(t)= \begin{cases}0, & t<t_{0} \\ r_{\mathrm{A}}^{*}(t), & t_{0} \leq t<t_{1} \\ 0, & t \geq t_{1} .\end{cases}$

Equation (15) implies that induced seismicity is fully explained by overloading, in agreement with the observation of no causal relationships between events in the Basel sequence (Langenbruch et al., 2011). The predicted rate (Eq. 15) and predicted cumulative number of events (Eq. 4) fit the data well, as shown in Fig. 3a and b, respectively. The role of temporal diffusion is observed after $t_{1}-\Delta t$ and is the only contributor to induced seismicity after $t_{1}$. Of three functional forms tested to describe diffusion (exponential, stretched exponential, and power law), the exponential (Eq. 14) was verified to be the best model for the Basel case (following the formalism and tests proposed by Clauset et al. (2009); see also Mignan $(2015,2016)$ for the tectonic aftershock case).

\section{Discussion}

The two descriptive laws of induced seismicity (one: linear relationship between fluid volume injected and cumulative number of events; two: parabolic spatial envelope) had been previously obtained by considering the differential equations of poroelasticity (Biot, 1941; Rudnicki, 1986) under a number of assumptions (Shapiro and Dinske, 2009). The algebraic expressions derived in the present study from geometric operations on a static stress field reflect a lower description length of the physical process (Kolmogorov, 1965) since all of Biot's theory is bypassed although similar characteristics of induced seismicity are modelled at the end (Fig. 4). Although the commonly used parabolic expression $r(t)=\sqrt{4 \pi D t}$, with $D$ being the hydraulic diffusivity and $t$ the time since the injection start (Shapiro et al., 1997), is relatively simple to derived from linear poroelasticity, it generally badly describes the early stage of the injection. This led to the addition of an arbitrary non-zero starting time $t_{0}$ in previous works (e.g. Shapiro et al., 2006), including the Basel case (Shapiro and Dinske, 2009), and finally to the consideration of non-linear poroelasticity (ibid.). The proposal in the present article of a more parsimonious and transparent approach obviously does not mean that it is superior to poroelasticity. It should simply be seen as a new alternative to induced seismicity modelling that is worth exploring in more detail.

The simplicity of the geometrical approach might a priori only appear applicable to homogeneous cases, such as the 2006 Basel EGS stimulation example. In fact, the approach could be applied to more problematic data sets that involve anisotropy and other heterogeneities. The most common example of anisotropy is the case of induced seismicity being spatially guided by a fault structure, such as during the 2004-2005 German Continental Deep Drilling Programme (KTB) injection (Shapiro et al., 2006) or the 2013 St Gallen, Switzerland stimulation (Edwards et al., 2015). Figure 5 illustrates how such heterogeneity can be implemented in the geometrical approach, by adding the historical static stress field that is associated with an active tectonic fault. It should be noted that this idea was first suggested in Mignan (2011) to explain the observed variability in tectonic precursory seismicity patterns. In the example of Fig. 5, a fault is located between $1.5<r<3.0$ away from the borehole $(r=0)$. If a mainshock occurred on that fault in the distant past, it would have created an overloading field (i.e. $\sigma>\sigma_{0}^{*}+\Delta \sigma^{*}$ ). Over time, this static stress field would have been "planed" to the 
Poroelastic approach

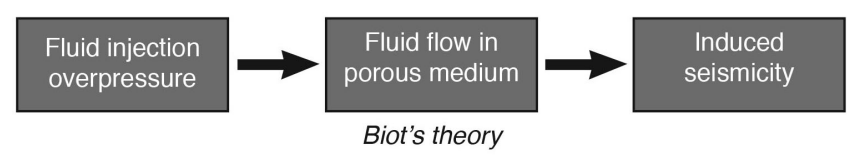

Geometrical approach

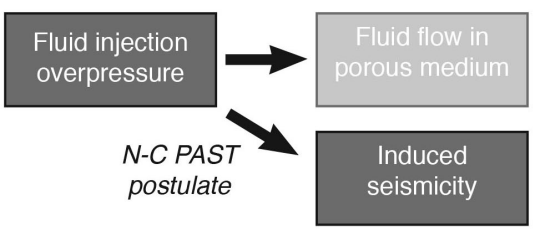

Description length

Figure 4. Description length defined as the count of physical steps required to describe induced seismicity, in poroelasticity and in the newly proposed geometrical approach. In the latter, Biot's theory is entirely bypassed.

threshold $\sigma_{0}^{*}+\Delta \sigma^{*}$ by temporal diffusion (represented by aftershocks, i.e. Eq. 14). This would yield a permanent "ghost" of that historical static stress field. It follows that during fluid injection there would then be two clusters of induced seismicity, one spherical, centred on the borehole, and a second, elongated, following the fault structure (Fig. 5).

\section{Conclusions}

I have demonstrated that the two principal induced seismicity descriptive laws can be explained from geometric operations in a static stress field without requiring any concept derived from poroelasticity. I have shown that the controlling parameter is then the normalised background stress amplitude range $\widehat{\Delta \sigma^{*}}$, which questions the usefulness of permeability and diffusivity parameters in induced seismicity analyses and might explain why these parameters remain elusive (Miller, 2015). In that view, permeability could depend on the "external loading configuration" instead of on the material itself, as recently proposed in the case of the static friction coefficient (Ben-David and Fineberg, 2011). Testing of the model on other induced seismicity sequences will determine if $\widehat{\Delta \sigma^{*}}$ is itself universal, region-specific, or related to the static stress memory of the crust, hence whether or not $\widehat{\Delta \sigma^{*}}$ depends on the tectonic loading configuration at EGS natural laboratory sites. Similar questions apply to the earthquake production parameter $\delta_{\mathrm{bp}}$ and if the two parameters are independent or correlated.

The main assumption of the N-C PAST is to consider three unique seismicity regimes (quiescence, background, and activation) defined by the event productions $\delta_{\mathrm{bm}}<\delta_{\mathrm{b} 0}<\delta_{\mathrm{bp}}$.

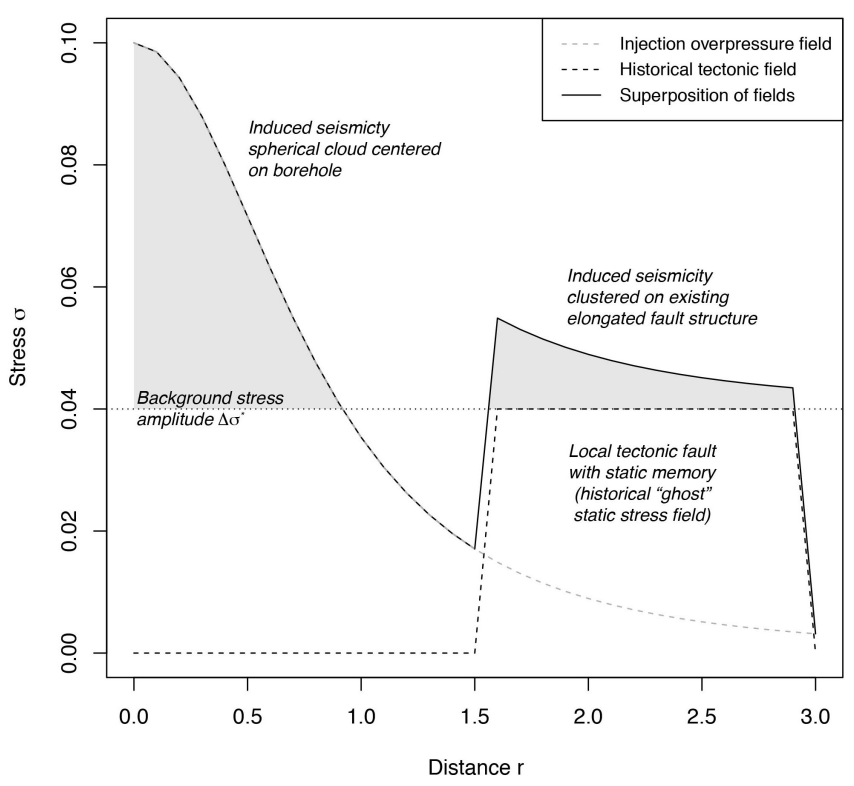

Figure 5. Sketch on how anisotropy and other types of heterogeneities can be implemented in the geometrical approach by adding a historical tectonic static stress field (ad hoc parameter values used for sake of simplicity). Here a past overloading field $\left(\sigma>\sigma_{0}^{*}+\Delta \sigma^{*}\right)$ on a nearby fault would have been "planed" to the threshold $\sigma_{0}^{*}+\Delta \sigma^{*}$ by temporal diffusion (Eq. 14), leaving only a "ghost" of that historical static stress field (for the homogeneous case, see Fig. 1a).

There are two possible physical alternatives to justify this choice: (1) it represents the fundamental behaviour of the Earth's crust, which would hence act as a capacitor, with strain energy storage and $\delta_{\text {bp }}$ analogues to electrical energy storage and capacitance, respectively (a parallel between tectonic aftershocks and a discharging Leyden jar is for instance made in Mignan, 2016); (2) the proposed step function is a simplification of the true stress-production profile, which remains unknown and is so far best characterised by three regimes (e.g. King, 2007). Both alternatives allow defining spatio-temporal solids over which geometric operations yield algebraic expressions of the induced seismicity behaviour.

Acknowledgements. I thank two anonymous reviewers for their comments. The work leading to this article was funded by the Swiss Competence Center for Energy Research - Supply of Electricity (SCCER-SoE).

Edited by: R. Gloaguen

Reviewed by: three anonymous referees 


\section{References}

Bak, P. and Tang, C.: Earthquakes as a Self-Organized Critical Phenomenon, J. Geophys. Res., 94, 15635-15637, 1989.

Ben-David, O. and Fineberg, J.: Static Friction Coefficient Is Not a Material Constant, Phys. Rev. Lett., 106, 254301, doi:10.1103/PhysRevLett.106.254301, 2011.

Berryman, J. G., and Wang, H. F.: Dispersion in poroelastic systems, Phys. Rev. E, 64, 011303, doi:10.1103/PhysRevE.64.011303, 2001.

Biot, M. A.: General Theory of Three Dimensional Consolidation, J. Appl. Phys., 12, 155-164, doi:10.1063/1.1712886, 1941.

Clauset, A., Shalizi, C. R., and Newman, M. E. J.: Power-Law Distributions in Empirical Data, SIAM Review, 51, 661-703, doi:10.1137/070710111, 2009.

Edwards, B., Kraft, T., Cauzzi, C., Kästli, P., and Wiemer, S.: Seismic monitoring and analysis of deep geothermal projects in $\mathrm{St}$ Gallen and Basel, Switzerland, Geophys. J. Int., 201, 1022-1039, doi:10.1093/gji/ggv059, 2015.

Ellsworth, W. L.: Injection-induced Earthquakes, Science, 341, doi:10.1126/science.1225942, 2013.

Gallier, J.: Curves and surfaces in geometric modeling, theory and algorithms, Morgan Kaufmann, Burlington, USA, 491 pp., 1999.

Grasso, J.-R. and Sornette, D.: Testing self-organized criticality by induced seismicity, J. Geophys. Res., 103, 29965-29987, 1998.

Häring, M. O., Schanz, U., Ladner, F., and Dyer, B. C.: Characterisation of the Basel 1 enhanced geothermal system, Geothermics, 37, 469-495, doi:10.1016/j.geothermics.2008.06.002, 2008.

King, G. C. P.: Fault interaction, Earthquake Stress Changes, and the Evolution of Seismicity, in: Treatise on Geophysics 4, Earthquake Seismology, edited by: Schubert, G., Elsevier, Amsterdam, 225-255, 2007.

Kolmogorov, A. N.: Three approaches to the quantitative definition of information, Problemy Peredachi Informatsii, 1, 3-11, 1965.

Kraft, T. and Deichmann, N.: High-precision relocation and focal mechanism of the injection-induced seismicity at the Basel EGS, Geothermics, 52, 59-73, doi:10.1016/j.geothermics.2014.05.014, 2014.

Langenbruch, C., Dinske, C., and Shapiro, S. A.: Inter event times of fluid induced earthquakes suggest their Poisson nature, Geophys. Res. Lett., 38, L21302, doi:10.1029/2011GL049474, 2011.

Majer, E. L., Baria, R., Stark, M., Oates, S., Bommer, J., Smith, B., and Asanuma, H.: Induced seismicity associated with Enhanced Geothermal Systems, Geothermics, 36, 185-222, doi:10.1016/j.geothermics.2007.03.003, 2007.

Mignan, A.: Non-Critical Precursory Accelerating Seismicity Theory (NC PAST) and limits of the power-law fit methodology, Tectonophysics, 452, 42-50, doi:10.1016/j.tecto.2008.02.010, 2008.
Mignan, A.: Retrospective on the Accelerating Seismic Release (ASR) hypothesis: Controversy and new horizons, Tectonophysics, 505, 1-16, doi:10.1016/j.tecto.2011.03.010, 2011.

Mignan, A.: Seismicity precursors to large earthquakes unified in a stress accumulation framework, Geophys. Res. Lett., 39, L21308, doi:10.1029/2012GL053946, 2012.

Mignan, A.: Modeling aftershocks as a stretched exponential relaxation, Geophys. Res. Lett., 42, 9726-9732, doi:10.1002/2015GL066232, 2015.

Mignan, A.: Revisiting the 1894 Omori aftershock dataset with the stretched exponential function, Seismol. Res. Lett., 87, doi:10.1785/0220150230, online first, 2016.

Mignan, A., King, G. C. P., and Bowman, D.: A mathematical formulation of accelerating moment release based on the stress accumulation model, J. Geophys. Res., 112, B07308, doi:10.1029/2006JB004671, 2007.

Mignan, A., Landtwing, D., Kästli, P., Mena, B., and Wiemer, S.: Induced seismicity risk analysis of the 2006 Basel, Switzerland, Enhanced Geothermal System project: Influence of uncertainties on risk mitigation, Geothermics, 53, 133-146, doi:10.1016/j.geothermics.2014.05.007, 2015.

Miller, S. A.: Modeling enhanced geothermal systems and the essential nature of large-scale changes in permeability at the onset of slip, Geofluids, 15, 338-349, doi:10.1111/gfl.12108, 2015.

Rudnicki, J. W.: Fluid mass sources and point forces in linear elastic diffusive solids, Mech. Mater., 5, 383-393, 1986.

Rutqvist, J.: Status of the TOUGH-FLAC simulator and recent applications related to coupled fluid flow and crustal deformations, Comput. Geosci., 37, 739-750, doi:10.1016/j.cageo.2010.08.006, 2011.

Sammis, C. G. and Sornette, D.: Positive feedback, memory, and the predictability of earthquakes, P. Natl. Acad. Sci. USA, 99, 2501-2508, doi:10.1073/pnas.012580999, 2002.

Shapiro, S. A. and Dinske, C.: Scaling of seismicity induced by nonlinear fluid-rock interaction, J. Geophys. Res., 114, B09307, doi:10.1029/2008JB006145, 2009.

Shapiro, S. A., Huenges, E., and Borm, G.: Estimating the crust permeability from fluid-injection-induced seismic emission at the KTB site, Geophys. J. Int., 131, F15-F18, 1997.

Shapiro, S. A., Kummerow, J., Dinske, C., Asch, G., Rothert, E., Erzinger, J., Kumpel, H.-J., and Kind, R.: Fluid induced seismicity guided by a continental fault: Injection experiment of 2004/2005 at the German Deep Drilling Site (KTB), Geophys. Res. Lett., 33, L01309, doi:10.1029/2005GL024659, 2006. 